\title{
LETTER
}

\section{A Pneumatic Device for Rapid Loading of DN A Sequencing Gels}

\section{Dimitrios A. Panussis, Mark W. Cook, Lisa L. Rifkin, Jacqueline E. Snider, Joseph T. Strong, Rebecca M. McGrane, Richard K. Wilson, and Elaine R. Mardis ${ }^{1}$}

\author{
Washington University, School of Medicine, Genome Sequencing Center, St. Louis, Missouri 63108 USA
}

This work describes the design and construction of a device that facilitates the loading of DNA samples onto polyacrylamide gels for detection in the Perkin Elmer/A pplied Biosystems (PE/ABI) 373 and 377 DNA sequencing instruments. The device is mounted onto the existing gel cassettes and makes the process of loading high-density gels less cumbersome while the associated time and errors are reduced. The principle of operation includes the simultaneous transfer of the entire batch of samples, in which a spring-loaded air cylinder generates positive pressure and flexible silica capillaries transfer the samples. A retractable capillary array carrier allows the delivery ends of the capillaries to be held up clear of the gel during loader attachment on the gel plates, while enabling their insertion in the gel wells once the device is securely mounted. Gel-loading devices capable of simultaneously transferring 72 samples onto the PE/ABI 373 and 377 are currently being used in our production sequencing groups while a 96 -sample transfer prototype undergoes testing.

The conventional method of loading multiple samples onto a horizontal or vertical slab-type electrophoresis gel is carried out by means of single- or multiple (typically eight)-tip hand-held pipettors. Of particular interest to our sequencing center is sample transfer onto Perkin Elmer/Applied Biosystems (PE/ABI) 373 and 377 DNA sequencers, because large numbers of these instruments are being used for daily DNA sequencing data production. Our increased genome sequencing efforts have been enabled by increases in the lane density of gels, the number of the DNA sequencing machines themselves, and the number of times per day each instrument is loaded. However, the task of manually loading gels becomes more time consuming, cumbersome, and error prone as the lane density increases to $>48$ samples per gel. Depending on the severity of the error, any remedy would take from a few hours to a day to correct, because in certain cases, the DNA samples need to be resequenced. Also, the manual loading of samples one at a time yields a time lag between the first and last samples that can result in diffusion-related band broadening. Hence, the effort to increase throughput requires reliable faster gel-loading methodologies.

${ }^{1}$ Corresponding author.

E-MAIL emardis@watson.wustl.edu; FAX (314) 286-1810.
We investigated currently available devices suitable for transferring DNA samples from the sample trays (0.2-ml thin-walled thermal cycling plate) into the corresponding wells. Apart from the hand-held single-tip pipettors, there are 6-, 8-, and 12-tip pipettors available. The latter could be used for the purpose of loading slab gels, provided that the pipettor's tip spacing matches both the well-to-well distance of the comb and the spacing of the sample tubes. An automated device, The GS Gene Loader II Automated Gel Loader (Bio-Rad 1992), was an instrument developed originally to transfer single samples from their source tubes into the designated well one at a time by use of an $x-y$ axis-mounted syringe. In addition, a peristaltic pumping system for sample transfer onto sequencing gels has been described (Smith and Watson 1993, 1994, 1995). In this approach, the DNA transfer tubes had smallbore capillary tubes attached at their ends for insertion into the wells of the gels. The transfer channels were individually driven, and the transfer lines themselves extended from the cart or bench holding the sample tray to the wells on the ABI gel. More recently (Erfle et al. 1997), the Biochemical Instrumentation Group at the European Molecular Biology Laboratory (EMBL) Heidel berg, Germany, developed a gel-loading technique using combs made of cellulose or nylon flexible membrane material. 
The user requirements in our case called for a type of device essentially different from the aforementioned devices offered. Our goal was to simultaneously transfer the entire batch of samples from the sample tray to the gel, and to outperform manual transfer with respect to time and consistency. Furthermore, the device must be easy to handle, clean, and maintain.

\section{RESULTS}

Once a prototype gel-loading unit was fabricated, we tested the device within our Technology Development Group to evaluate its performance and to obtain user input with regard to hardware performance and suggested design modifications. These were incorporated into a second prototype, which was further eval uated by a subset of our production team members. We finalized the design following this second evaluation and proceeded with the manufacture of an adequate number of production units. Newer versions of the gel-loader reflect the increasing gel-loading demands as a result of the increasing scale of production sequencing. Historically, four 64-lane gel-loading units for the PE/ABI 373 DNA sequencer had been in daily service with in our production sequencing groups from January 1996 until June 1997. At that time, twelve 72-lane units were introduced for use on the $29 \mathrm{PE} / \mathrm{ABI} 373$ sequencers. In October 1997, sixteen 72-lane gel loaders for the $45 \mathrm{PE} / \mathrm{ABI} 377$ sequencers were completed and now are in daily production use. Recently, we introduced the colorless perforated version of the 96-well tray (1038-64-0A; Robbins Scientific, Sunnyvale, CA), and are currently testing this plate to optimize its use with the 72-lane gel loaders. This plate allows us to precipitate the combined, completed sequencing reactions into a 96-well format plate rather than into the more expensive strips of $0.2-\mathrm{ml}$ thermal cycling tubes. On loading, the sample tray segment containing the $G$ and $H$ rows is detached at the perforation. The location of a second perforation between the plain edge and the $\mathrm{H}$ row, also removed upon loading, allows three remnant G-H tray segments from three different sets of sequencing reactions to be placed side by side in the sample tray rack and loaded onto a fourth gel.

The availability of more sensitive detection systems coupled with improvements in the labeling chemistry (Ju et al. 1995a,b) have allowed overall cost savings by reducing reaction volumes. Currently, the targeted sample volumes to load manually are $\sim 2 \mu \mathrm{l}$ on both the PE/ABI 373 and 377 se- quencers. When the gel loaders are used, the initial sample volume in the tubes is $3 \mu \mathrm{l}$ of a standard formamide loading solution ( $80 \%$ formamide, 8.3 mM EDTA, $0.67 \%$ blue dextran). The target sample volume ranges from 1.0 to $1.2 \mu \mathrm{l}$, that is, the approximate capacity of the shorter wells in our staggered well-forming comb for the PE/ABI 377 sequencer. Starting with $3 \mu \mathrm{l}$ allows the technician to hand load any missed wells.

Because our development and fabrication of the gel-loading devices was supported entirely by federal grants, the gel-loading technology is available to other U.S. Iaboratories also supported by federal grants. Washington University has applied for U.S. and international patents on the gel loader concept and design. On the basis of our experience, we are willing to assist other research laboratories in building their own gel loading units, provided the transferred technology will not be used for commercial purposes.

\section{DISCUSSIO N}

The measurable benefits of using the gel-loading devices in our production sequencing operation have been an overall reduction in the number of people required to load gels and in the number of sample placement errors in the gel-loading process. In the case of the PE/ABI 377 instrument, in which visibility of the sample wells is poor and manually loading the DNA samples is more difficult, we have been able to achieve high-density gels with minimal difficulty. Overall, the number of persons required to load each group's five ABI 373 sequencers with 64 samples per gel was reduced by one (from three to two) per load, whereas the increased accuracy of sample placement on gels is more difficult to measure. Indexing fixtures for each type of sequencing instrument were developed to assist the gel loading technician in hand loading any missed wells that may result from clogged or broken capillaries.

Capillary replacement on gel loaders is a commonly voiced concern. During the 4 months from June to September 1997, 291 capillaries were replaced on our production 72-lane 373 gel loaders. Overall, 1.65 capillaries are replaced per active gel loader per week of usage. Because the PE/ABI 373 sequencers are run twice a day on each week day and once each day during the weekend days, the capillary cost per sequencing instrument per run is $\sim 0.138$ capillaries, or 18.6 cents/run, on the basis of the latest capillary acquisition cost. Capillary replacement averages $\sim 5$ min per capillary and is per- 
formed by an experienced technician in our group because of the required precision of placement. The fabrication cost and time for the gel-loading device required 6 weeks to mill and assemble for the latest batch of 72-lane PE/ABI 377 gel loader units and cost $\sim \$ 1600$ per unit for labor and purchased parts.

Increased sample capacity to allow loading of an entire 96-sample batch is the next development step for both the PE/ABI 373 and 377 gel loaders. This increase entails redesign of the existing staggered well-forming combs to cast 96 wells across the same laser detection distance and upgrading the existing gel loaders to transfer 96 samples. This work will be coordinated with our progress in increasing the pixel density of gel images for DNA sequencing instruments as mentioned previously. The concept of device-assisted gel loading as described here for $\mathrm{PE} / \mathrm{ABI}$ sequencing instruments can potentially be applied to other DNA sequencers and gel types with appropriately modified configurations.

\section{METHODS}

\section{Specifying the Gel-Loading Process}

The number of DNA samples loaded per sequencing gel have increased steadily over the past few years as a result of improvements to DNA sequencing instruments. At the same time, a number of our current projects aim to increase further the detection capability of the existing DNA sequencing instruments beyond their present density of 72 samples per gel. Minimizing pressure losses along the transfer lines requires that the distance between the sample tray and the gel wells is minimized, and that no sudden changes in the cross section of the flow occur. The latter implies a uniform transfer line without attachments on the delivery or intake ends. Any reduction in the transfer distance for DNA samples will decrease the loading times and the amount of unused sample remaining inside the transfer lines. The idea of using capillaries as transfer conduits stemmed from the fact that capillaries would be thin enough to insert into the gap between the two glass plates containing the gel. Furthermore, capillaries coated externally with a plastic material are flexible enough to use in one continuous piece throughout the length of the transfer line from intake to delivery. A suitable capillary for insertion in both the 373 and 377 gels is composed of flexible fused silica, comprising a glass core of 100 $\mu \mathrm{m}$ inner diameter, with an external polyimide coating of $170 \mu \mathrm{m}$ outside diameter, providing strength and flexibility (TSP100170; Polymicro Technologies, Inc., Phoenix, AZ).

\section{Pressure Chamber Design}

The pressure chamber consists of three parts (Fig. 1) and is designed to allow for expansion to 96 lanes with minimal modifications. The main aluminum body defines the chamber space and essentially contains the sample tubes held in the sample tube rack. The sample tubes can be either in the form of strips of $0.2-\mathrm{ml}$ thin-walled tubes or of a standard 96-tube tray (Robbins Cycleplate). The main body is capped with a white delrin plate (Fig. 1), which holds an array of seventy-two 0.5-inch-long stainless-steel (gauge 24) hypodermic receptor tubes. The latter are press-fit in the 9-mm centerto-center format of a standard 96-well sample tray. A groove is milled on the top surface of the main aluminum body to contain an O-ring (2-157; Sealtite Corp., St. Louis, MO). The delrin cap plate is bolted down on top of the main pressure chamber piece, and the enclosed O-ring seals the interface. In the assembly phase, the intake ends of the capillaries are passed through the receptor tubes, registered flush with the flat bottom face of the pressure chamber, and glued in place to the guide tube with a droplet of fast-acting cyanoacrylate glue. An increase to 96-lane capability involves uncapping the pressure chamber, removing the previously set capillaries, drilling 24 additional receptor holes on the delrin cap plate, and recapping the chamber with the modified plate. Then a set of 96 new capillaries is installed.

The lower aluminum carriage plate delivers samples into the main chamber by sliding along a stainless-steel rod. This plate also includes a shallow pocket to contain the sample tube rack so that tube centers remain aligned with the capillaries. The pocket depth is set to position the capillary intake ends at the bottom of each sample tube. Thus, the entire sample contents can be picked up and dispensed. A groove is milled around the shallow pocket to contain a red silicone

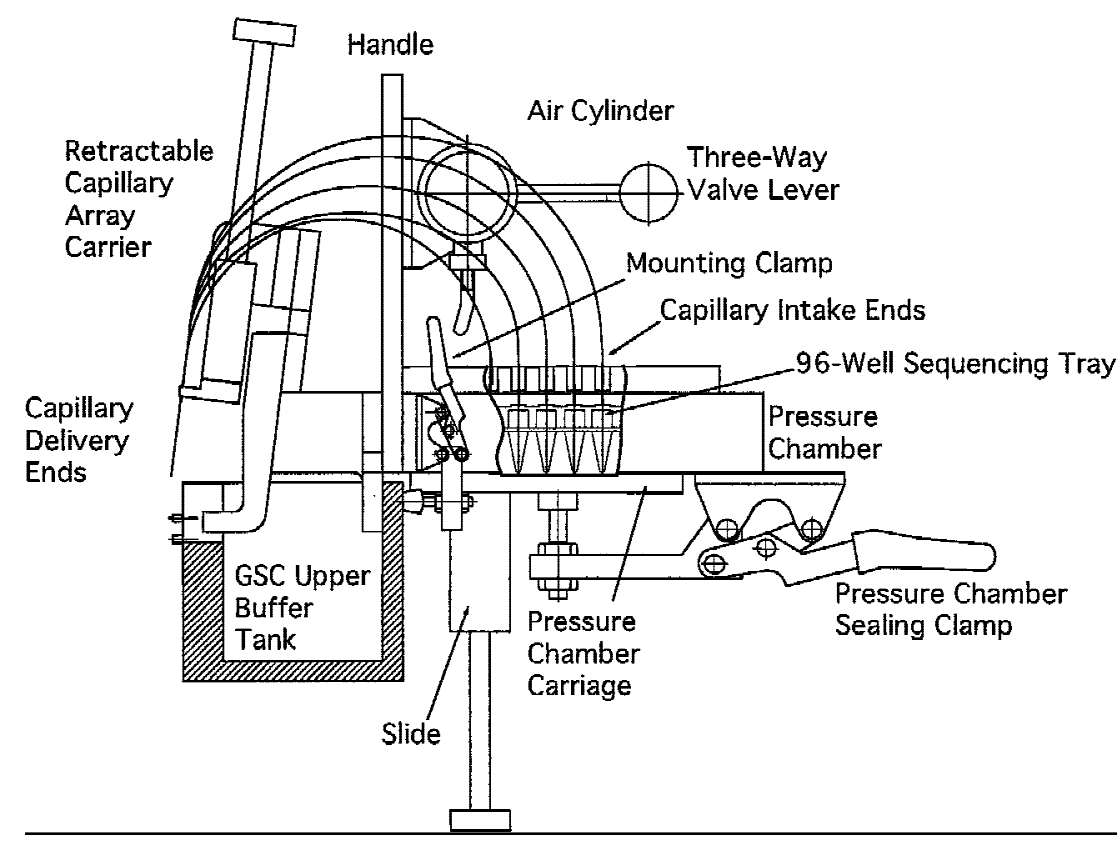

Figure 1 Schematic of the gel loader for the PE/ABI 377 sequencer, showing the main components of the device. The gel loader is mounted onto the Genome Sequencing Center upper buffer tank. To maintain clarity, only five capillaries and a partial cutout of the pressure chamber are shown. 
O-ring (2-160; Sealtite), which seals the pressure chamber when the plate is up against the main chamber. This assembly is clamped by means of two stainless-steel hold-down action clamps (215-USS; Destaco Co., Troy, MI). The delrin rack holding the sampletubes was designed to accommodate strips of tubes (1044-50-0; Robbins Scientific Corp., Sunnyvale, CA), as well as the 96-well sequencing tray (1038-00-1, -2, -3, -4; Robbins Scientific), used in manual gel loading.

Small-bore-size spring-loaded air cylinders equipped with a manually operated three-way valve were used to pressurize the main pressure chamber. We have selected a 11/16inch bore size, 3-inch stroke model, for use on the 72-lane gel loading units (SR-093-M; Bimba Manufacturing Company, Monee, IL). This pneumatic device is used as a hand-operated air pump and is permanently mounted on the acrylic cover of the gel-loading unit itself. Silicone rubber tubing (51135K 72 ; McM aster-Carr, Chicago, IL) connects the pressure port of the air cylinder to the main pressure chamber port. The connection occurs via two brass el bow pipe fittings (44555K145; McMaster-Carr), one attached to the air cylinder and one to the main pressure chamber. A miniature plastic tube coupling (51605K12; M cMaster-Carr) and an in-line body (51602K14; McM aster-Carr) separate the two halves of the silicone rubber tubing, which allows for the ease of connect/disconnect of air cylinder from pressure chamber during maintenance. Pressurizing the gel loader occurs by first extending the piston rod of the air cylinder out all the way with the three-way valve in the open position. The valve is then switched to the closed position and the piston rod is released. Because the extended spring inside the cylinder tries to return to its initial rest position, the air inside the pressure chamber is compressed so that a positive pressure develops and forces the DNA samples through the capillaries.

\section{The Retractable Capillary A rray}

The capillaries must be protected from accidental breakage during handling and storage. Although much of the exposed length of the capillaries can be covered with a protective shield most of the time, the delivery ends are more vulnerable during gel loading. On the initial 64-lane gel loader model for the $\mathrm{PE} / \mathrm{ABI} 373$, the capillary array was fixed onto the instrument body. Here, the delivery ends of the capillaries were inserted into the gel wells at the same time as the gel loader unit was mounted onto the glass plates, which often resulted in capillary breakage. On the subsequent 72-lane models for both the PE/ABI 373 and 377 sequencers, a retractable capillary array has replaced the fixed one. The 373 version makes use of a low-profile ball slide (BA1-05LP; Design Components, Inc., Franklin, MA). The capillary array carriage attached to the slide is locked in the up (retracted) position by means of two stainless-steel ball plungers (34085A89; M cM aster-Carr). The latter engage into two depressions, which are milled on the sliding capillary carriage. The slide on the 377 version utilizes a stainless-steel rod (S4-45; W.M. Berg, Inc., East Rockaway, NY), which slides in a bronze sleeve bearing (6391K135; M cM aster-Carr) (Fig. 1). The capillary array carriage attached to the slide is locked in the upper (retracted) position by means of a stainless-steel spring plunger (84985A77; M cM aster-Carr). The latter engages into a groove, which is milled on the sliding rod of the carriage. During the period from January 1996 to June 1997, 2670 capillaries were replaced on the four 64-lane $\mathrm{PE} / \mathrm{ABI} 373$ gel-loading units then in service, each having a fixed capillary array. This corresponds to 10.4 replaced capillaries per active gel loader per week of usage. By comparison, only 1.65 capillaries per active gel loader per week were replaced during the first 4 months of usage of the 72-Iane retractable capillary array version of the 373 loader.

\section{Interfacing the Gel Loader with the PE/ A BI 373 Sequencer}

The upper buffer tank of the PE/ABI 373 sequencer is mounted behind the notched rear glass plate, al lowing the gel loader to be directly mounted onto the front glass plate. The capillary array holder approaches the gel gap between the two glass plates at an angle of $7^{\circ} \mathrm{C}$ to facilitate capillary insertion. The delivery tips of the capillaries are aligned, not staggered, and the insertion depth is $1 / 8$ inch. The gel loader is mounted on the front glass plate by means of two delrin spacers, held against the rear face of the glass plate with two stainless-steel hold-down action clamps (205-USS; Destaco). The clamps carry rubber-tipped spindles (105-208; Destaco) to permit a firm but flexible mounting. Vertically, the del rin spacers support the gel loader on the ridge of the rear notched plate. A del rin bar, mounted on the two spacers, runs across the capillary array and shields the delivery end of the capillaries.

To ensure proper alignment between the capillaries and the wells in the gel, a new well-forming comb was designed and fabricated. The gel spacers and comb are made from Kynmar polyester gauge 0.014 inch $(\sim 0.36 \mathrm{~mm}$ thick; Cope Plastics, Inc., St. Louis, MO). The center-to-center distance between the wells is 0.0785 inch, with a wall thickness of 0.0250 inch and a capacity of $0.49 \mu \mathrm{l} / \mathrm{mm}$ of well height. The polyester comb is adhered to an acrylic backing bar, which extends in one aspect beyond the comb's end. When inserted into the unpolymerized gel, the comb is placed so that this extension contacts the right notch of the glass plate. The backing bar also ensures that the teeth are consistently set to the same depth. Thus, the comb is registered with reference to the inner corner of the right notch. The center of the rightmost well in the gel is the same distance from the right notch, as is the center of the rightmost capillary on the gel loader from the outer edge of the right del rin spacer. When the gel loader is mounted on the gel plates, the right spacer is positioned with its right edge against the inner side of the right plate notch. Thus, both the gel loader and the well-forming comb are registered from the same reference point horizontally and vertically. The delrin part, holding the array of capillary delivery tips, bears mounting slots instead of holes, which are used to align the array in the final step of gel-loader assembly.

\section{Interfacing the Gel Loader with the PE/ A BI 377 Sequencer}

On the PE/ABI 377 sequencing instrument, the upper buffer tank and the notched glass plate are on the front of the gel cassette, a difference that introduces a number of issues concerning the mounting and alignment of the gel loader. First, there is no space behind the rear glass plate available to mount the gel loader, as on the 373 model. Second, the manufacturer's upper buffer tank does not represent a reliable hardware interface between our gel-loading device and the gel plates. Thus, we designed and fabricated a new upper buffer 


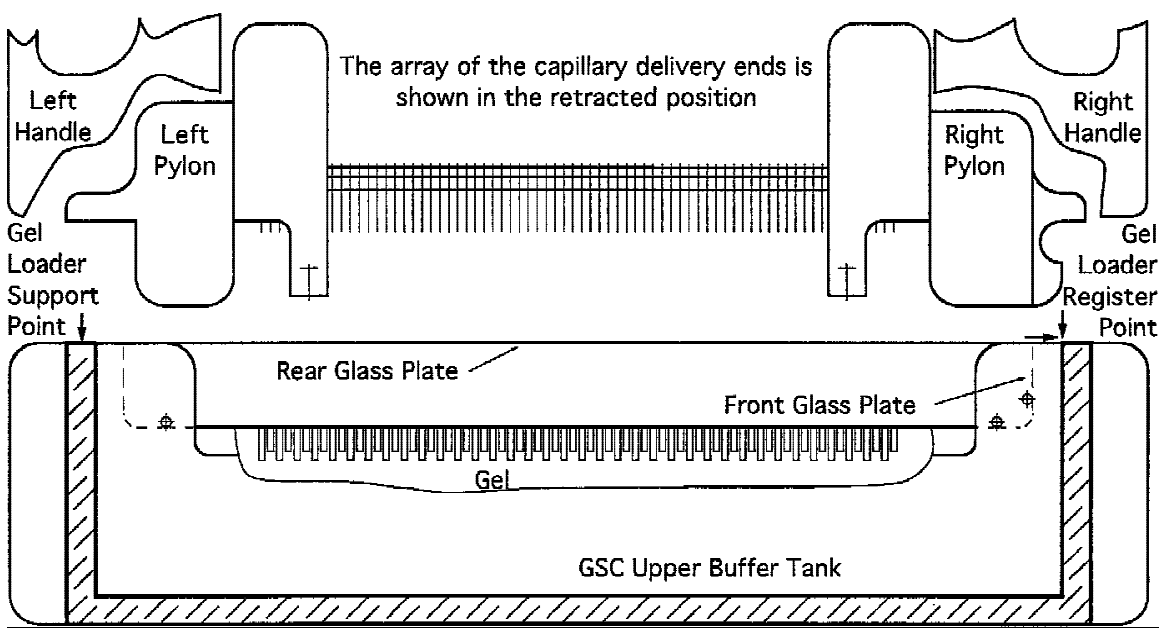

Figure 2 For the purpose of alignment, both the well-forming comb and the upper buffer tank for the PE/ABI 377 sequencer are registered off the right notch of the front glass plate. The gel loader is registered off the right inner wall of the upper buffer tank.

tank that replaces the manufacturer's version exactly. Three 3/32-inch-diam. stainless-steel pins are press-fit into the upper buffer tank body to assist in locating and registering of the tank from the inner corner of the front glass plate's right notch (Fig. 2). The approximate cost to machine and assemble this buffer tank is $\$ 283$ per unit for a batch size of 60 units.

The gel loader is mounted on the front panel of the upper buffer tank by the two stainless-steel hold-down action clamps (205-USS; Destaco) and is referenced off the inside face of the tank's right wall (Figs. 2 and 3). Vertically, the gel loader rests on the ridge of the tank's front wall panel. Thus, the gel loader is referenced, through the upper buffer tank, off the right notch of the front glass plate. Two ear-like acrylic extensions, attached to the acrylic cover of the device and engaged with the uppermost set of clamps on the gel cassette, further support the weight of the gel loader. On the 377 loader, the capillary array approaches the gel in the direction opposite to that on the 373 model, with the angle of insertion maintained at $7^{\circ} \mathrm{C}$.

The rear of the acrylic cover contains and shields the capillaries when they are in the retracted position. To ensure proper alignment between the capillaries and the wells in the gel, a new 72-lane well-forming comb for the 377 model has been designed and fabricated. The material of the gel spacers and the comb itself is Kynmar polyester gauge 0.0075 inch ( $0.19 \mathrm{~mm}$ thick; (Cope Plastics). The center-to-center distance between the wells is the same as in the 373 model (0.0785 inch), with a wall thickness of 0.0250 inch and a capacity of $0.26 \mu \mathrm{l} / \mathrm{mm}$ of well height. The acrylic backing bar extension is placed opposite that on the 373 model (Fig. 2), to compensate for the reverse gel-plate orientation on model 377 and to maintain the same direction of referencing. The inner edge of the right plate notch is the common reference for locating the rightmost gel well as well as the inner right wall of the upper buffer tank (Fig. 2). Thus, both the gel loader and the well-forming comb are registered from the same reference point horizontally and vertically. Calibration of the capillary array on final assembly is as described for the 373 loader.

\section{Preparing the A crylamide Gels}

High quality of well formation on DNA sequencing gels is critical to successful sample loading when using the gel loader and also extends the operational life of the capillaries. The introduction of the retractable capillary array has reduced the incidence of tips becoming clogged with polyacrylamide gel during the gelloading process. Proper registration of the gel loader and well-forming comb during gel pouring ensures that the capillaries will drop directly into the gel wells. Gel formation is dependent on two factors: (1) the application of bind silane solution to the inner surfaces of both gel plates prior to assembly; and (2) the smooth removal of the comb from the polymerized gel so as to avoid damage to the wells. Any excess gel remaining in the cutout area of the notched glass plate must be washed out with warm water. Detailed procedures describing the glass plate preparation and gel pouring have been described (Wilson and Mardis 1997).

\section{Procedure for A utomatic Gel Loading}

With the gel-loading unit on its supporting stand, the wash tray is half filled with deionized water and placed in the pressure chamber carriage. The latter is slid up and clamped, and

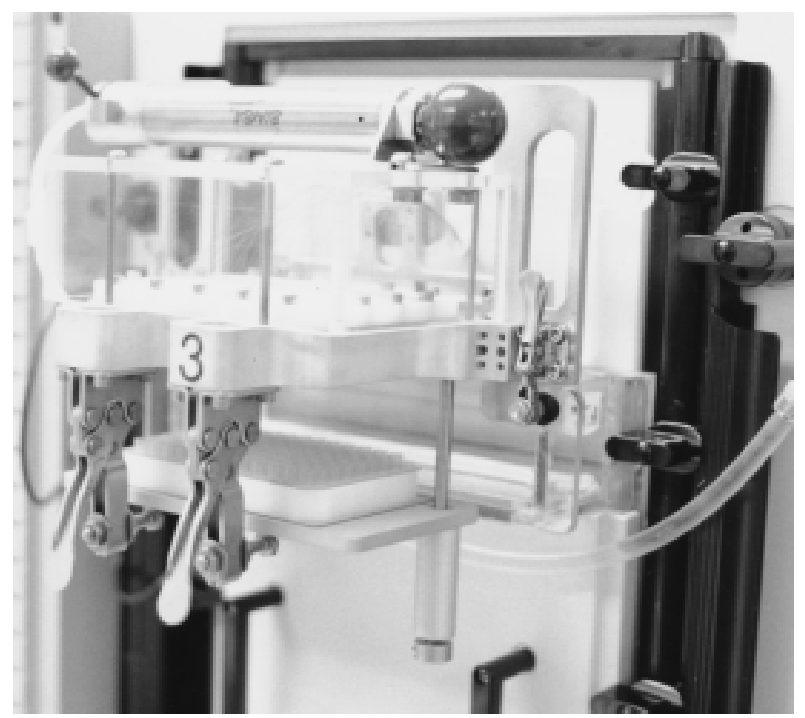

Figure 3 A 72-lane gel loader mounted on the upper buffer tank and the gel cassette of the PE/ABI 377 sequencer. The Genome Sequencing Center upper buffer tank is shown mounted on the PE/ABI instrument. The gel-loading device is attached onto the upper buffer tank. 
the system is pressurized. Once all of the capillaries are primed, water droplets form at the tips. The system pressure is released and the pressure chamber unclamped. The wash tray is replaced with the sample rack that contains either strip tubes or a 96-well plate of DNA samples, each resuspended in $3 \mu \mathrm{l}$ of loading dye. The pressure chamber is again clamped and the unit is mounted onto the gel cassette with the retractable capillary array in the retracted position. The unit is registered off the right glass notch and clamped securely into place. The capillary array is lowered, which inserts the capillaries into the wells to the predetermined depth. The operator pressurizes the system via the spring-loaded air cylinder and this initiates sample transfer. Observation of sample transfer by the operator determines when to interrupt the process by releasing any remaining pressure. Once sample transfer is completed, the capillary array is retracted. The gel loader is removed and placed back onto the support stand. The pressure chamber is unclamped, and the sample rack is replaced with the water-filled wash tray to flush the capillaries clean of any remaining dye in the transfer lines prior to loading the next gel. The cycle time for the procedure above is $\sim 5 \mathrm{~min}$ per gel. Once a gel-loading session is completed, the gel loader is rinsed as above and any remaining water is removed from the capillaries by pressurizing the chamber with air only. Capillaries are dry when stored, with the carriage plate unclamped in the lower position.

The steps followed with the gel loader are less manipulative than those with manual gel loading. Using the gel loader requires attention to detail during mounting of the device, during lowering of the retractable capillary array, and when the sample transfer is terminated. In contrast, manual gel loading requires the technician's attention as each sample is transferred into each one of the wells. The 6.7-lb. weight of the gel-loading device allows for ease of transport from the stand onto the gel cassette and back.

\section{M aintaining the Gel-Loader U nits}

The capillaries on the gel loader must be flushed clean of any residual DNA sample after each loading, as described above. Additionally, gel loader parts that were exposed to TBE buffer, including the delivery ends of the capillaries and the spacers or pylons being used to mount the unit, must be rinsed off. Clogged capillaries are replaced by disconnecting the tube between the air cylinder and the pressure chamber and then removing the acrylic cover from the pressure chamber body. The two stainless-steel guide tubes holding the damaged capillary are extracted from the delrin pressure chamber cover. Then, two new guide tubes are press-fit with pliers into the receptor holes and a new capillary is passed through the guide tubes with the ends aligned to the appropriate height both inside the pressure chamber and on the capillary array. A droplet of fast-acting cyanoacrylate glue joins the outer capillary wall to each guide tube.

\section{A CKN O WLEDG MENTS}

We thank Dr. Robert H. Waterston, James S. McDonnell Professor and Chairman of the Department of Genetics at the Washington University School of Medicine, for his constant support and encouragement. We also thank John Kreitler, Manager of the Instrument Machine Shop at the Washington University Medical Center, for his assistance in fabricating the in-house designed components and assembling the gelloading units. Also, we thank the personnel of the Genome Sequencing Center for their comments and suggestions for modification and improvement of these devices during the testing period. Their interest in putting the gel loaders in daily use was the greatest motivation for the gel-loader project. This work has been supported by National Institutes of Health/ National Human Genome Research Institute (NIH/NHGRI) Grant 5P01HG0095604: Large-Scale Genome Sequencing, and grant 5P50HG0145802: The Human Genome Sequence-a Pilot Project.

The publication costs of this article were defrayed in part by payment of page charges. This article must therefore be hereby marked "advertisement" in accordance with 18 USC section 1734 solely to indicate this fact.

\section{REFEREN CES}

Bio-Rad. 1992. GS Gene Loader II automated gel loader user's guide. Bio-Rad Laboratories, Life Sciences Group, Richmond, CA.

Erfle, H., R. Ventzki, H. Voss, S. Rechmann, V. Benes, J. Stegemann, and W. Ansorge. 1997. Simultaneous loading of 200 sample lanes for DNA sequencing on vertical and horizontal, standard and ultrathin gels. Nucleic Acids Res. 25: $2229-2230$.

Ju, J., C. Ruan, C.W. Fuller, A.N. Glazer, and R.A. Mathies. 1995a. Fluorescence energy transfer dye-labeled primers for DNA sequencing and analysis. Proc. Natl. Acad. Sci.

92: 4347-4351.

Ju, J., I. Kheterpal, J.R. Scherer, C. Ruan, C.W. Fuller, A.N. Glazer, and R.A. Mathies. 1995b. Design and synthesis of fluorescence energy transfer dye-labeled primers and their application for DNA sequencing and analysis. Anal.

Biochem. 230: 131-140.

Smith, A. and A. Watson. 1993. Sequencing gel loader. GB patent no: 9321650.5 .

1994. Parallel loading of sequencing gels. Sixth International Genome Sequencing and Analysis Conference, Hilton Head Island, SC.

1995. Sequencing gel loader. International patent no: PCT/GB94/02297.

Wilson, R.K. and E.R. Mardis. 1997. Fluorescence-based DNA sequencing. In Genome analysis: A laboratory manual. Volume I "Analyzing DNA" (ed. B. Birren, E.D. Green, S. Klapholz, R.M. Myers, and J. Roskams), pp. 301-395. Cold Spring Harbor Laboratory Press, Cold Spring Harbor, NY.

Received November 10, 1997; accepted in revised form March 13, 1998. 


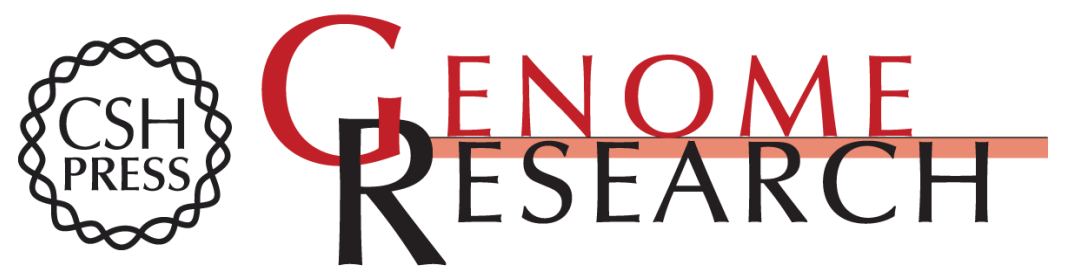

\section{A Pneumatic Device for Rapid Loading of DNA Sequencing Gels}

Dimitrios A. Panussis, Mark W. Cook, Lisa L. Rifkin, et al.

Genome Res. 1998 8: 543-548

Access the most recent version at doi:10.1101/gr.8.5.543

$\begin{array}{ll}\text { References } & \text { This article cites } 2 \text { articles, } 1 \text { of which can be accessed free at: } \\ \text { http://genome.cshlp.org/content/8/5/543.full.html\#ref-list-1 }\end{array}$

License

Email Alerting Receive free email alerts when new articles cite this article - sign up in the box at the Service top right corner of the article or click here.

\section{Affordable, Accurate} Sequencing.

To subscribe to Genome Research go to: https://genome.cshlp.org/subscriptions 